\title{
Has dengue found its home in Hong Kong?
}

\author{
${ }^{1}$ CM Poon, BSSC, ${ }^{2}$ SS Lee *, MD, FHKAM (Medicine) \\ ${ }^{1}$ Jockey Club School of Public Health and Primary Care and ${ }^{2}$ Stanley Ho Centre for Emerging Infectious Diseases, \\ The Chinese University of Hong Kong, Hong Kong
}

Hong Kong Med J 2015;21:85-7

*sslee@cuhk.edu.hk

This article was published on $5 \mathrm{Dec}$ 2014 at www.hkmj.org.
In Hong Kong, the recent report of locally acquired dengue cases has raised concern about the introduction of dengue virus in our community. Within just over a month, three people with no travel history during the incubation period have been confirmed to have dengue virus infection. As there was no epidemiological link between the third patient and the previous two, multiple sources of infection and the possibility of ongoing transmission within Hong Kong are suggested. Temporally, the emergence of these three cases follows closely the large outbreak in Guangdong, which affected more than 40000 people. Given the scale of the outbreak, the Department of Health of Guangdong Province has released updates on the dengue situation on a daily basis from 22 September 2014 to the end of
October 2014. ${ }^{1}$ Dengue activity peaked between 29 September and 13 October, with almost 10000 cases reported each week. More than $80 \%$ of the cases were notified in Guangzhou.

Epidemiological information for the Guangdong outbreak can be accessed on the Internet, which allows us to map the dengue distribution by city and the changes over time with the Geographic Information System. The scale of the outbreak has evidently expanded geographically in Guangdong since its onset, as shown by the biweekly standard deviational ellipses, each of which would have covered approximately $68 \%$ of the dengue cases in the respective fortnight (Fig). Guangzhou remained the spatial centre of the outbreak during these weeks. A similar temporal distribution of dengue cases was

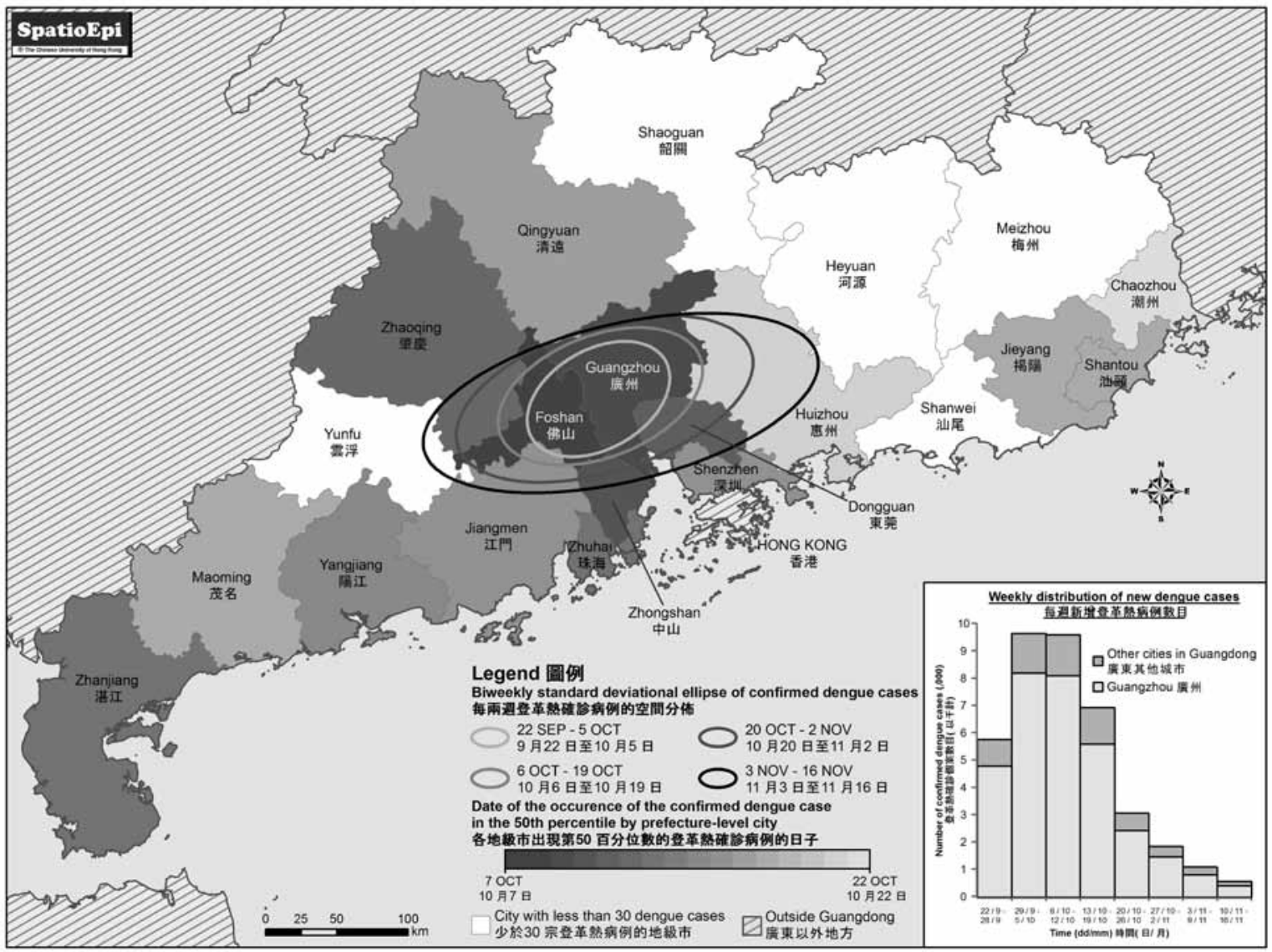

FIG. Spatiotemporal distribution of confirmed dengue cases between 22 September and 16 November 2014, from data released by the Department of Health of Guangdong Province published in the daily dengue report 
observed in other cities in the Pearl River Delta (PRD), while places further away from the delta were subsequently affected at a diminished level, like a ripple effect. Researchers from Mainland China had previously suggested the PRD and Chao Shan Area as the two main hubs in Guangdong where indigenous dengue cases have clustered since the turn of the century. ${ }^{2}$ It appears, therefore, that this latest outbreak is the result of extensive transmission of dengue virus from the PRD hub.

Even before the change in sovereignty, the PRD was gradually transformed into a Chinese region where many inhabitants move within and between cities every day. With the rising number of people who live in the PRD, Hong Kong is no different to a prefecture-level city of Guangdong. Currently, Hong Kong's population comprises an estimated 200000 mobile residents, with the majority travelling back and forth to Guangdong. ${ }^{3}$ Mainland China's transport system, with its increasing efficiency, not only reduces the travel time between cities in Guangdong, but also accelerates the propagation of infections in the region. In the past decade, dengue outbreaks have been reported in places as far apart as Zhejiang and Henan, ${ }^{4-6}$ a scenario proving that human mobility is crucial in predisposing to virus spread. It is conceivable that the latest Guangdong outbreak has led to concurrent maintenance of multiple pools of actively infected people commuting in the PRD who, in the presence of the Aedes mosquitoes, have enabled the virus to find a new home base. Beginning in August 2014, three local dengue cases were reported in $\mathrm{Macau}^{7}$ coinciding with the reported onset of the Guangdong outbreak. The Guangdong epidemic undoubtedly increases the likelihood of exposure to dengue virus among mobile residents, the magnitude of which varies with the place, time, and duration of their stay, as well as their commuting frequency. Geographically, dengue virus transmission might be more likely to occur in some districts in Hong Kong. This does not necessarily imply a direct association with the size of the vector population, but the heterogeneous distribution of mobile residents in the territory. ${ }^{3}$

Theoretically, one useful way to guard Hong Kong from importation of dengue virus is to track the situation in neighbouring countries. However, the uniqueness of the natural history of dengue virus infection poses a challenge to accurately predicting its epidemiological risk. Clinically, dengue fever is typically a self-limiting condition with an incubation period ranging between 3 and 14 days. ${ }^{8}$ Since most people infected with dengue virus have no or mild signs or symptoms, a significant proportion are never diagnosed or reported. ${ }^{9}$ Consequently, estimation from the size and distribution of actively infected people can hardly reflect dengue epidemiology. Serological testing of dengue antibody may infer the proportional distribution of the population with previous exposure to the virus, but is unable to determine the actual size of the 'infective' population. A study in 2007 to 2009 in Hong Kong gave a prevalence of positive dengue immunoglobulin G of $1.6 \%$, a figure that cannot provide insight into the current epidemiological status. ${ }^{10}$ Importantly, clinical testing plays no direct role in public health interventions. It is also almost impossible to intercept asymptomatic travellers or those in latent infection from entering Hong Kong. Introduction of dengue virus into our neighbourhood is therefore often silent and discovered after several waves of transmissions.

Upon identification of a locally acquired dengue case in Hong Kong, epidemiological investigations and vector control measures have been conducted immediately to prevent secondary spread. Coincidentally, the three recently reported local dengue patients either worked or lived in the vicinity of a construction site, and breeding of mosquito larvae was found during site inspection. ${ }^{11}$ This reminds us of the lesson from the outbreak related to a construction site in Ma Wan in 2002, affecting 16 workers and residents nearby. ${ }^{12}$ Whereas the previous outbreak was limited to an island, the residences and suspected infection sources of the three recent local dengue patients were sporadically distributed in several densely populated districts, suggesting a higher risk for subsequent evolvement of dengue endemicity. Prior to the Ma Wan outbreak, a major dengue outbreak occurred in Macau in the preceding year, with a total of 1418 reported cases. ${ }^{13}$ Although the exact source of the Macau outbreak could not be ascertained, it also occurred at a time when infrastructure development was gaining momentum. Dengue dissemination could well be the outcome of a perfect match between increasing human mobility and urban development. Understandably vector surveillance is a key component of current environmental measures. Ovitraps have been strategically established in 44 locations in Hong Kong to monitor the extensiveness of the distribution of Aedes mosquitoes. ${ }^{14}$ However, the insufficient geographical coverage of ovitraps limits their ability to detect the invasion of dengue virus as Aedes albopictus has a short flight range of no more than 200 metres. ${ }^{15}$ Thus, the eggs sampled in the ovitraps can only represent the mosquitoes in a confined geographic area. Nevertheless, the importance of eliminating mosquito breeding sites in our highly urbanised environment cannot be overemphasised. This is, however, a real challenge, even for dengue-endemic Singapore where the hygiene standard is enviably high. ${ }^{16}$

Even without any new locally acquired dengue cases in the coming months, the medical profession should remain vigilant and advise on 
effective prevention. The virus can hide in the eggs of infected mosquitoes this winter and becomes reactivated when the weather is favourable for breeding again. ${ }^{17}$ The next round of local dengue transmission could probably begin after the spring, merging with the anticipated upsurge of indigenous dengue infections in the PRD every late summer, ${ }^{2}$ which are our potential external sources of dengue virus. In consideration of the incident local dengue cases and our dengue endemic neighbours, there is no doubt that dengue virus has found its home in Hong Kong. It is just a matter of whether this is a temporary home or a permanent base.

\section{References}

1. The Department of Health of Guangdong Province. Updates on epidemics [in Chinese]. Available from: http:// www.gdwst.gov.cn/a/yiqingxx/. Accessed 18 Nov 2014.

2. Li Z, Yin W, Clements A, et al. Spatiotemporal analysis of indigenous and imported dengue fever cases in Guangdong province, China. BMC Infect Dis 2012;12:132.

3. Census and Statistics Department, Hong Kong. 2011 Population Census. Available from: http://www. census2011.gov.hk/en/index.html. Accessed 18 Nov 2014.

4. Xu G, Dong H, Shi N, et al. An outbreak of dengue virus serotype 1 infection in Cixi, Ningbo, People's Republic of China, 2004, associated with a traveler from Thailand and high density of Aedes albopictus. Am J Trop Med Hyg 2007;76:1182-8.

5. Wu JY, Lun ZR, James AA, Chen XG. Dengue fever in mainland China. Am J Trop Med Hyg 2010;83:664-71.

6. Huang XY, Ma HX, Wang HF, et al. Outbreak of dengue fever in central China, 2013. Biomed Environ Sci 2014;27:894-7.

7. Health Bureau, Macao. Infectious disease information.
Available from: http://www.ssm.gov.mo/portal/csr/ch/ main.aspx. Accessed 27 Nov 2014.

8. Rudolph KE, Lessler J, Moloney RM, Kmush B, Cummings DA. Incubation periods of mosquito-borne viral infections: a systematic review. Am J Trop Med Hyg 2014;90:882-91.

9. Chastel C. Eventual role of asymptomatic cases of dengue for the introduction and spread of dengue viruses in nonendemic regions. Front Physiol 2012;3:70.

10. Lo CL, Yip SP, Leung PH. Seroprevalence of dengue in the general population of Hong Kong. Trop Med Int Health 2013;18:1097-102.

11. Hong Kong SAR Government press release. Government enhances anti-mosquito measures in prevention of dengue fever. 21 November 2014. Available from: http://www. info.gov.hk/gia/general/201411/11/P201411110853.htm. Accessed 27 Nov 2014.

12. Ma SK, Wong WC, Leung CW, et al. Review of vectorborne diseases in Hong Kong. Travel Med Infect Dis 2011;9:95-105.

13. Almeida AP, Baptista SS, Sousa CA, et al. Bioecology and vectorial capacity of Aedes albopictus (Diptera: Culicidae) in Macao, China, in relation to dengue virus transmission. J Med Entomol 2005;42:419-28.

14. Audit Commission, Hong Kong. Director of Audit's Report No. 63. Available from: http://www.aud.gov.hk/pdf_e/ e63ch05.pdf. Accessed 24 Nov 2014.

15. Marini F, Caputo B, Pombi M, Tarsitani G, della Torre A. Study of Aedes albopictus dispersal in Rome, Italy, using sticky traps in mark-release-recapture experiments. Med Vet Entomol 2010;24:361-8.

16. Ooi EE, Goh KT, Gubler DJ. Dengue prevention and 35 years of vector control in Singapore. Emerg Infect Dis 2006;12:887-93.

17. Lee HL, Rohani A. Transovarial transmission of dengue virus in Aedes aegypti and Aedes albopictus in relation to dengue outbreak in an urban area in Malaysia. Dengue Bulletin 2005;29:106-11. 